\title{
Mobile genetic elements in antibiotic resistance
}

Chromosomally-mediated resistance, arising from mutation during therapy or spread of a single organism, can be a major contributor to the resistance encountered in bacterial infection. Chromosomal mutations may change membrane permeability, reducing the efficacy of many antibacterial agents, induce the production of chromosomally encoded enzymes such as $\beta$-lactamases, or alter the target enzyme, e.g., dihydrofolate reductase (DHFR), the binding site of trimethoprim. The nature of these mechanisms is such that they can only be passed on vertically to the progeny of the resistance organisms. Thus, they account for only a small proportion of resistant isolates and generally mediate only lowlevel resistance. Plasmid-mediated resistance accounts for most high-level resistance found in bacteria and has resulted in the rapid spread and accumulation of the most efficient resistance mechanisms with a concomitant challenge to antibacterial therapy.

The evolution and spread of resistance plasmids has been promoted by the wide use of antibiotics in medicine and animal husbandry. In the presence of antibiotics, the acquisition of a resistance plasmid by the host cell is obviously beneficial. It will survive and multiply, as will the plasmid, whilst non-plasmid carrying cells will be eliminated. Plasmids are passed on vertically to the cell's progeny during cell division. However, many resistance plasmids are also capable of mediating their transfer to other organisms, and indeed to organisms of other species, by conjugation. This greatly increases the potential for the spread of resistance genes situated on plasmids. The plasmid, which is separate from the cell in evolutionary terms, ensures its maintenance in the cell whether or not antibiotic selection pressure is present. Resistance plasmids seem to be stable in bacterial populations, ${ }^{1}$ even when there is no selective pressure; indeed their stability will benefit the progeny of the cell in future encounters with the antibiotic, which may be likely in the case of commonly used drugs.

Most resistance plasmids mediate resistance to more than one, sometimes as many as seven, antibiotics. ${ }^{2}$ Selective pressure applied by the use of a single antibiotic will select for all the other resistance mechanisms. ${ }^{3}$ Many outbreaks of plasmid-mediated resistance causing problems in hospital or clinical environments have been documented and single or closely related plasmids have often been shown to be responsible. ${ }^{4}$

How have these problematic and promiscuous organelles evolved? Plasmids may have originated from an aberrant phage of from the excision of a piece of chromosome containing an origin of replication with the subsequent acquisition of the genes we see today. This was, however, a very ancient event. Resistance plasmids are similar to plasmids found in strains of gram-negative rods isolated before the antibiotic era that do not carry resistance mechanisms, indicating that resistance genes must have been acquired since the introduction of antibiotics into medicine. ${ }^{5}$ How have plasmids accumulated such diversity of resistance genes? Some plasmids are not essential for bacterial survival under normal circumstances; they are free to mutate without lethal effects on the cell. They can also move between cells and species, accumulating genes by recombination. Homologous recombination, integration and excision from the host chromosome must play some part in the acquisition of genes by plasmids. However, transposition has been shown increasingly to be a major contribution to plasmid development since the first recognition of a transposon, $\mathrm{Tn} 1$, by Hedges and Jacob. ${ }^{6}$ Complete and functional resistance genes can be transferred from one replicon to another by this mechanism.

Two major classes of transposon have been identified; class I or composite transposons and class II or complex transposons differ in structure and function. They may originate from the use of modified restriction endonucleases or recombinases which have acquired functions in recognition and recombination of specific sequences. Class I transposons are a heterogeneous group of elements, carrying a variety of resistance genes that share similar structural and functional properties, but share little DNA homology. ${ }^{7}$ The class II transposons are more homogeneous, comprising three different but related "families", the Tn $3, \operatorname{Tn} 21$ and Tn2501 groups. ${ }^{8.9}$ Transposon 21 and its group have been the most widely studied. These transposons usually carry more than one resistance determinant $;,{ }^{910}$ resistance to sulphonamide is usually present; the OXA and PSE $\beta$-lactamases are common ${ }^{8}$ as is enzymatic resistance to the aminoglycoside antibiotics. ${ }^{11}$ Resistance to mercury compounds ${ }^{12}$ and trimethoprim resistance mediated by $\mathrm{dhfr}$ II and $\mathrm{V}$ are also associated with $\operatorname{Tn} 21 .{ }^{13}$ The dhfr I gene which is usually associated with $\mathrm{Tn} 7$ has also been shown as part of a Tn21-like transposon. ${ }^{14}$

It is not immediately obvious how such a homogeneous group of elements can harbour such a wide range of resistance mechanisms but it has been proposed that these structures were formed by the integration of discrete resistance elements into existing transposons. ${ }^{10}$ In these transposons, and plasmids that 
carry similar resistance mechanisms, e.g. R388 and $\mathrm{R} 46$, highly conserved regions are found in sequences immediately surrounding the structural gene. Flanking the structural gene are GTTA sequences which mark the insertion points for the resistance gene and represent recombination hot-spots necessary for crossover of resistance genes. ${ }^{15}$ At the 5 -end of the resistance gene there is an open reading frame which codes for a protein that shares homology with both transposon resolvases and phage site-specific integrases. ${ }^{16}$ This integrase has been designated int or $\operatorname{tnp} I^{16.17}$ and is responsible for the recombination of the resistance genes. At the 3 -end of the resistance gene is an imperfect palindromic region of $59 \mathrm{bp}$ that is essential for control of integration and contains the GTTA sequence which marks the point of excision. ${ }^{15}$

A gene "cassette", containing the resistance gene, the GTTA sequence and all but the last seven bases of the 59-bp region, has been proposed as the unit of recombination. ${ }^{18}$ Circularisation of the element, after excision from its original location, precedes its integration in the new replicon. ${ }^{18}$ This small mobile genetic element has been called an integron (In). ${ }^{16}$ The GTTA and an incomplete 59-bp sequence have been identified in $\mathrm{Tn} 7$ carrying the dhfrI gene. This gene has also been characterised in $\operatorname{Tn} 21$ as part of an integron, indicating an exchange between the two transposons. ${ }^{14}$ Evidence of an ancestral integron has been found in the Pseudomonas aeruginosa plasmid pVS1; this consists only of the integrase region and a single GTTA cross-over point. ${ }^{19}$ It has been proposed that the sulphonamide resistance gene (sulI) was initially integrated into this region at the 3 -end of the integrase. This reflects the early clinical use of the sulphonamides, since the sul gene is almost completely conserved in these structures. This has been followed by site-specific integration, mediated by the integrase, of multiple resistance genes in the form of "cassettes" and the 59-bp region at the original GTTA cross-over point which has formed the modern integron. Other recombination events, such as homologous recombination, will have influenced the evolution of the integrons to produce the diversity which is now apparent. ${ }^{19}$

Plasmids are responsible for widespread problems in the treatment of infections and have increasingly become the bete-noir of antibiotic chemotherapy. Transposons have certainly contributed to the evolution of resistance plasmids, providing genetic variation and allowing the dissemination of accumulated genes between plasmids and their incorporation into the chromosome. ${ }^{20}$ The integron has been shown to be responsible for the dramatic success of the Tn21-like transposons. Whether they are more widely involved in the evolution of plasmids and transposons is yet to be confirmed, although $\mathrm{Tn} 7$ has been shown to contain similar elements. The evolution of the antibiotic resistance mechanisms themselves, and the various means by which they are distributed has been driven by the selective pressure of antibiotic use. The evol- ution of the integron, which has so far only been implicated in antibiotic resistance, seems also to have been the result of antibiotic pressure. The use of many different antibiotics has resulted in the accumulation of multiple resistance mechanisms on single plasmids resulting in co-selection of different resistance mechanisms. The evolution of transposons, and perhaps integrons, allows flexibility in the genetic response to a battery of antibiotics.

S. TAIT

Department of Medical Microbiology, The Medical School, University of Edinburgh, Teviot Place, Edinburgh

\section{References}

1. Bennett PM, Linton AH. Do plasmids influence the survival of bacteria? J Antimicrob Chemother 1986: 18 Suppl C: $123-126$.

2. Foster TJ. Plasmid-determined resistance to antimicrobial drugs and toxic metal ions in bacteria. Microbiol Rev 1983; 47: $361-409$.

3. Amyes SGB, McMillan CJ, Drysdale JL. Transferable trimethoprim resistance amongst hospital isolates. In: Grassi GG, Sabath LD (eds) New trends in antibiotics: research an therapy. New York, Elsevier/North-Holland Biomedical Press. 1981: 325-327.

4. Amyes SGB, Doherty CJ, Young H-K. High-level trimethoprim resistance in urinary bacteria. Eur J Clin Microbiol 1986; 5: 287-291.

5. Datta N, Hughes VM. Plasmids of the same Inc groups in Enterobacteria before and after the medical use of antibiotics. Nature 1983; 306: 616-617.

6. Hedges RW, Jacob AE. Transposition of ampicillin resistance from RP4 to other replicons. Mol Gen Genet 1974; 132: 31-40.

7. Schmitt R. Molecular biology of transposable elements. $J$ Antimicrob Chemother 1986; 18 Suppl C: 25-34.

8. Lafond M, Couture F, Vézina G, Levesque RC. Evolutionary perspectives in multiresistance $\beta$-lactamase transposons. $J$ Bacteriol $1989 ; 171$ : 6423-6429.

9. Wiedemann B, Meyer JF, Zühlsdorf MT. Insertions of resistance genes into Tn2l-like transposons. I Antimicrob Chemother 1986; 18 Suppl C: 85-92.

10. Martinez E, de la Cruz F. Transposon Tn 21 encodes a RecAindependent site-specific integration system. Mol Gen Genet 1988; $211: 320-325$.

11. Wohlleben W, Arnold W, Bissonnette L et al. On the evolution of the $\operatorname{Tn} 21$-like multiresistance transposons: sequence analysis of the gene (aacCl) fo the gentamicin acetyltransferase-3-1 [AAC (3)-1], another member of the Tn21based expression cassette. Mol Gen Genet 1989; 217: 202-208

12. Brown NL, Misra TK, Winnie JN, Schmidt A, Seiff M, Silver S. The nusleotide sequence of the mercuric resistance operons of plasmid R100 and transposon Tn501; further evidence for mer genes which enhance the activity of the mercuric ion detoxification system. Mol Gen Genet 1986; 202: $143-151$

13. Sundström L, Rådström $P$, Swedberg G, Sköld O. Site-specific recombination promotes linkage between trimethoprimand sulfonamide-resistance genes. Sequence characterization of $\mathrm{dhfrV}$ and sulI and a recombination active locus of Tn21. Mol Gen Genet 1988; 213: 191-201.

14. Sundström L, Sköld $O$. The dhfrI trimethoprim resistance gene of $\operatorname{Tn} 7$ can be found at specific sites in other genetic surroundings. Antimicrob Agents Chemother 1990; 34 642-650.

15. Martinez E, De la Cruz F. Genetic elements involved in Tn2l site-specific integration, a novel mechanism for the dissemination of antibiotic resistance genes. EMBOJ 1990;9 : 1275-1281.

16. Stokes RW, Hall RM. A novel family of potentially mobile 
DNA elements encoding site-specific gene-integration functions: integrons. Mol Microbiol 1989; 3: 1669-1683.

17. Mercier J, Lachapelle J, Couture F et al. Structural and functional characterization of tnpI, a recombinase locus in Tn 21 and related $\beta$-lactamase transposons. $J$ Bacteriol $1990 ; 172$ : 3745-3757.

18. Collis CM, Hall RM. Site-specific deletion and rearrangement of integron insert genes catalyzed by the integron DNA integrase. J Bacteriol 1992; 174: 1574-1585.
19. Bissonnette L, Roy PH. Characterization of InO of Pseudomonas aeruginosa plasmid pVS1 an ancestor of integrons of multiresistance plasmids and transposons of gram-negative bacteria. J Bacteriol 1992; 174: 1248-1257.

20. Steen R, Sköld O. Plasmid-borne or chromosomally mediated resistance by $\mathrm{Tn} 7$ is the most common response to ubiquitous use of trimethoprim. Antimicrob Agents Chemother 1985; 27: 933-937. 